\title{
LAS AGUAS PLUVIALES Y DE TORMENTA: DEL ABANDONO DE UN RECURSO HÍDRICO CON FINALIDAD AGRÍCOLA A SU IMPLANTACIÓN COMO RECURSO NO CONVENCIONAL EN ÁMBITOS URBANOS ${ }^{1}$
}

\author{
María Hernández Hernández \\ Departamento de Análisis Geográfico Regional y Geografía Física \\ (Universidad de Alicante) \\ David Saurí Pujol \\ Departamento de Geografía (Universidad Autónoma de Barcelona) \\ Enrique A. Moltó Mantero \\ Departamento de Análisis Geográfico Regional y Geografía Física \\ (Universidad de Alicante)
}

\section{RESUMEN}

Las aguas de turbias desempeñaron un papel fundamental en los paisajes agrarios tradicionales del sureste peninsular. En ellos, la escasez de precipitaciones unido a su torrencialidad, obligó a la adopción de una sistematización de laderas y barrancos para aprovechar al máximo los caudales circulantes asociados a precipitaciones de cierta intensidad. Estos sistemas, que van a entrar en un proceso de abandono y desarticulación a partir de mediados del siglo $\mathrm{xx}$, han sido objeto de un interés creciente vinculado a cuestiones ambientales y patrimoniales. A éstas se une recientemente su posible utilización como recursos no convencionales asociados a las políticas de sostenibilidad ambiental y de adecuación de la calidad de las aguas según su uso (fit for purpose). El

1. Este artículo se inserta en los proyectos de investigación Urbanización y metabolismo hídrico en el litoral de Alicante: análisis de tendencias para el periodo 2000-2010 (CSO201236997-C02-02) y Análisis de los cambios recientes en el consumo de agua en entornos de urbanización acelerada: propuesta metodológica y estudio de caso para el litoral Mediterráneo español (CSO2012-36997-C02-01) financiados por el Ministerio de Economía y Competitividad. 
objetivo de este artículo es examinar, teniendo en cuenta el significado patrimonial y ambiental que estos recursos de agua desempeñaron en las sociedades agrarias tradicionales, su recuperación, desde el punto de vista de iniciativas a escala local y de sostenibilidad ambiental. Para ello se analizan las actuaciones llevadas a cabo en ámbitos como los municipios litorales de la provincia de Alicante y el área metropolitana de Cataluña.

Palabras clave: turbias, aguas pluviales, sostenibilidad, recursos no convencionales, Agenda local 21, Alicante, Barcelona

\section{ABSTRACT}

Rainwater: from abandonment for agricultural purpose to its implementation as a non-conventional resource in urban areas

Stormwater played a fundamental role in the traditional agricultural landscapes of Southeastern Spain. Relatively rare but violent outbursts of precipitation forced traditional agriculture to build a sophisticated system of slope and ravine management to take advantage of flows associated with intense rainfall. These systems, which will be entering a process of abandonment and disbandment from the middle of the 20th century onwards, have been the subject of growing interest linked to environmental and landscape conservation issues. On the other hand, rainwater flows also regain their importance in urban areas as local, alternative resources able to provide for the needs of certain uses such as garden irrigation after the concept of fit for purpose. The aim of this paper is to examine, initiatives aiming at the recovery of rainwater flows under the same philosophy of traditional agrarian societies but this time directed to the urban sphere. To this effect we will present cases in the coastal municipalities of the province of Alicante and the metropolitan area of Barcelona where rainwater and other alternative water flows are increasingly used.

Keywords, stormwater, sustainability, non-conventional resources, local Agenda 21, Alicante, Barcelona

\section{INTRODUCCIÓN}

El significado de las aguas de turbias en las sociedades agrarias tradicionales

En una sociedad como la tradicional, donde la agricultura era la base económica, suelo y agua adquirían particular relevancia. Por ello, cuando el medio no oferta, en la medida suficiente esos elementos, intentar mantener uno y acrecentar el otro, era la máxima que guió las actuaciones antrópicas en las tierras semiáridas del sureste peninsular. Éstas se sintetizaban en una doble aptitud: de una parte, buscar los medios para aprovechar las lluvias, generalmente concentradas en unos cuantos acontecimientos de la estación otoñal y, de otra, controlar su capacidad erosiva sobre los suelos y adoptar las medidas necesarias para reducir los coeficientes de escorrentía que se generan en los diferentes lechos y, de este 
modo, atenuar la pérdida de horizontes edáficos superficiales y más meteorizados (Hernández y Morales, 2013: 106-107)

El agricultor, para minimizar los rasgos citados, ideó una sistematización del territorio que es el resultado de una doble necesidad: disponer de tierras para el cultivo y suministrar a las mismas caudales, al menos en una cantidad que permita asegurar las cosechas, lo que no siempre resulta factible. Ello fue posible mediante la adopción de sistemas que permitían captar los volúmenes circulantes por las ramblas y barrancos coincidiendo con aguaceros copiosos; pero, también, otros orientados a la retención de la escasa humedad caída directamente sobre las parcelas o superficies aledañas a ellas a mayor cuota altimétrica. Para ello, se derivan los caudales circulantes con ocasión de aguaceros intensos, las denominadas aguas de turbias; llegándose de este modo a realizar lo que se conoce como inundación dirigida o cosecha de agua (Martínez de Azagra, 1996). Mediante la inducción y «recolección» de la escorrentía que discurre por laderas, vertientes y cauces, se lograba almacenar la máxima cantidad de agua que fuera posible en el suelo durante los meses relativamente húmedos (otoño, invierno y primavera). Posteriormente, mediante la realización de diversas labores del suelo, se reducía la evapotranspiración por capilaridad (Mondejar, 2010). De este modo, se conseguía conservar la mayor cantidad posible de humedad y durante el máximo tiempo posible en los perfiles profundos del suelo e incrementar las disponibilidades hídricas para los cultivos. Laderas, vertientes, lomas, barrancos y cauces fueron organizados y ordenados de manera que las escorrentías se pudieran conducir y derivar hacia las parcelas de cultivo situadas en cotas inferiores (Morales, 1969; Morales y Box, 1986).

Este conjunto de actuaciones permitió la ordenación de grandes superficies, que en el caso del sureste peninsular implicó la casi totalidad del territorio donde se practicaba el cultivo pluvial secano. El sistema de riegos de turbias creó lo que algunos autores denominaron como «secano mejorado» (López Gómez, 1951), es decir, se configura como un tipo de aprovechamiento intermedio entre el secano y el regadío, ya que esos mayores aportes hídricos permitían reducir la aleatoriedad del primero, pero en ningún caso consentían el desarrollo de cultivos de regadío en sentido estricto.

La escorrentía de laderas montañosas, e incluso de barrancos, se captaba y conducía, asimismo, mediante pequeñas boqueras para acumular el agua circulante en aljibes, balsas y estanques (Box, 1995). Con los primeros, se pretendía almacenar el agua necesaria para la población que vivía en estos territorios durante todo el año. Con las segundas, se atendía temporalmente a las necesidades hídricas de los ganados y animales de tracción. Los terceros se utilizaban para los mismos fines de las segundas y, además, para irrigar pequeños huertos, que abastecían temporalmente de hortalizas a los habitantes de estos poblamientos rurales dispersos 
Del abandono y degradación de estos paisajes a su «redescubrimiento» desde la óptica patrimonial y ambiental

Estas técnicas, asociadas a unos paisajes agrícolas y también puntualmente para el abastecimiento, van a registrar un progresivo abandono a partir de mediados del siglo veinte. Éste es resultado de varios factores. El primero, la baja rentabilidad de los cultivos de secano que no pudieron resistir la competencia salarial surgida en la costa, donde se instalan actividades secundarias y terciarias a mediados del siglo veinte, así como áreas de cultivo de regadío que producen frutos extratempranos de elevada cotización en los mercados nacionales y extranjeros; proceso que se traduce en una significativa reducción de los activos agrarios a los que hay que unir un importante éxodo rural. El segundo, va a ser la posibilidad de disponer de mayores volúmenes de agua, bien mediante la regulación de las escasas disponibilidades autóctonas, una mejor administración y reparto de los mismos, o la obtención de aportes subterráneos gracias a la utilización de bombas de gran capacidad e incluso procedentes de otras cuencas (Hernández y Morales, 2013).

El abandono de estos terrazgos agrícolas y la omisión de determinadas tareas conllevan la progresiva destrucción de toda la sistematización. Éste no sólo se va a traducir en la desarticulación de estos paisajes, unas veces por abandono y otras por la implantación de sistemas de cultivo que no contaban con la incidencia de los fuertes aguaceros sobre los nuevos parcelarios, sino, también, la pérdida de las ventajas que esta ordenación generaba y, consiguientemente, la acentuación de aquellos procesos que minimizaban como era, entre otros, la laminación de las crecidas dada la considerable reducción de los coeficientes de escorrentía y una enmienda orgánica y natural del suelo (Moltó y Hernández, 2012). Se conseguía con ello laminar y mermar los volúmenes de las crecidas y, en definitiva, una aminoración de los destructivos efectos de las avenidas fluviales en los tramos bajos. Igualmente, se creaban suelos agrícolas con la retención de légamos e incrementando la infiltración en los suelos, la recarga de acuíferos. De manera que, ahora cuando se producen chubascos de fuerte intensidad horaria, la arroyada superficial se ve acelerada en relación a su funcionamiento en los siglos anteriores, provocando un aumento considerable de los caudales circulantes y, lógicamente, potenciando la capacidad erosiva de estas ramblas (Marco y Vera, 1988; Morales, 1989; Marco y Morales, 1995). Resultado de todo lo indicado anteriormente es que las corrientes generadas por estos aguaceros adquieren una elevada capacidad de carga. Las consecuencias de esta situación se han dejado sentir no sólo allí donde estos fenómenos se han desencadenado, sino, como es lógico también en las partes más bajas de las cuencas vertientes. A los procesos de degradación 
ambiental e incremento de los riesgos naturales, se une la degradación paisajística y patrimonial.

Paralelamente, a estos procesos de pérdida de saberes relacionados con la gestión del territorio y la intensificación de las actividades agrarias y la difusión de los usos urbano-residenciales en amplios territorios que poseían altos grados de naturalidad y/o ruralidad, en los últimos treinta años se ha producido una serie de corrientes que apuestan por una recuperación de estas ordenaciones (Hernández y Moltó, 2010; Hernández y Olcina, 2013). Éstas iniciativas se vinculan con diversos procesos. Una primera corriente se relaciona con el «redescubrimiento» de los paisajes culturales. En este proceso desempeñan un papel fundamental los valores que estas sociedades les asignan, a saber, económico, ambiental y cultural. El primero, se vincula al hecho de que además de producir materias primas, ofertan servicios relacionados con la difusión de las actividades turístico-residenciales. El segundo, relacionado con la creciente preocupación por su degradación del paisaje, al ser considerado como un recurso natural más; por lo que es contemplado, con mayor frecuencia, por amplias capas sociales como un bien escaso, difícilmente renovable y fácilmente degradable. El tercero, relacionado con elementos patrimoniales, culturales y de identidad, al ser reflejo de las relaciones entre una sociedad y su entorno (Hernández, 2009). Estas iniciativas en gran medida se asocian con las políticas de desarrollo rural y de diversificación de las actividades económicas vinculadas a la conservación de estos paisajes.

Una segunda corriente, con los beneficios ambientales generados por esta ordenación del territorio. Diferentes colectivos (conservacionistas, técnicos medioambientales, agentes de desarrollo local de las administraciones, entre otros), se han planteado en las últimas dos décadas hasta que punto sería factible la restauración y el mantenimiento de los antiguos sistemas de aterrazamientos y riegos por boqueras, desde el punto de vista de las ventajas que aportan al medio cultural respetuoso con el físico, entre las que cabe citar conservación de suelo, recarga de los acuíferos, laminación de las ondas de crecida, construcción de depósitos para la lucha contra incendios (Generalitat Valenciana, 2003), la repoblación forestal (Mogil y Martínez de Azagra, 2006), etc.

Una tercera corriente y relacionada con la anterior es la que contempla estos recursos circulantes coincidiendo con precipitaciones de fuerte intensidad horaria como un recurso de agua no convencional y cuyo uso se insertaría en actuaciones que abogan por la sostenibilidad de los usos del agua.

\section{OBJETIVOS, MÉTODO DE TRABAJO Y ÁREA DE ESTUDIO}

Una utilización del medio basada en un conocimiento profundo del espacio y su dinámica natural fue lo que permitió adaptarse a las condiciones imperantes 
en el sureste español y, con ello, aprovechar al máximo cuanto del medio era posible, contribuyendo, a la vez, a controlar las arroyadas, pues la ruptura de pendientes por el abancalamiento, al tiempo que la desviación de caudales por las boqueras, implicaba una considerable reducción de los coeficientes de escorrentía y una enmienda orgánica y natural del suelo. A estos valores, su une el significado que pueden adquirir en las sociedades actuales donde las demandas de agua son crecientes y, en ámbitos donde por factores físicos y de demanda son escasos.

Teniendo en cuenta este planteamiento, los objetivos de esta publicación son:

a. poner de manifiesto la importancia creciente que, desde una óptica teórica, han adquirido los recursos de agua no convencionales en el marco de sistemas que abogan por la sostenibilidad (por ejemplo, las Agendas locales 21) y por el concepto de calidad de agua vinculada al uso al que van destinadas («fit for purpose»).

b. considerar desde una óptica territorial, la posible recuperación de estos sistemas basados en el aprovechamiento de aguas pluviales en entornos urbanos vinculados a la gestión de la demanda y con sistemas más sostenibles,

c. analizar la incorporación de este tipo de recursos en entornos urbanos en dos áreas de estudio, a modo de estudio de caso; poniendo de manifiesto los puntos fuertes y débiles de este proceso.

El análisis de la literatura relativa, por un lado, a la ordenación tradicional del sureste peninsular donde se analiza su génesis, evolución y estado actual $\mathrm{y}$, por otro, a recursos no convencionales y su implementación al amparo de actuaciones en el marco de políticas más sostenibles ha permitido elaborar un estado de la cuestión concerniente a ambas temáticas, pero también poner de manifiesto las interrelaciones que se establecen entre ambas cuestiones, tanto desde una óptica teórica como de potencial aplicación. A partir de ella, ha sido posible llevar a cabo un estudio de las iniciativas que han apostado por la incorporación de este tipo de recursos. Para ello, se ha consultado las agendas locales 21 al ser considerada esta herramienta como el instrumento a partir del cual se pueden adoptar actuaciones más sostenibles en el marco de la gestión de la demanda y de los recursos. En una tercera fase, se analiza de manera pormenorizada las actuaciones implementadas en dos ámbitos territoriales, a modo de estudio. Este examen multiescalar permite poner de manifiesto cuales son los ámbitos de mayor implantación, así como los puntos fuertes y débiles de estas iniciativas.

El litoral mediterráneo español representa un espacio particularmente interesante para explorar los ciclos hidrosociales vinculados con aguas desalinizadas, 
pluviales y residuales. La elección de dos áreas de estudio (área metropolitana de Barcelona y municipios litorales de las comarcas del Campo de Alicante y Vega Baja del Segura) no es baladí, responde a una serie de principios básicos, a saber:

a. elección de un territorio (municipios litorales de las comarcas del Campo de Alicante y Vega Baja del Segura) donde por factores físicos la escasez de recursos determinó que la ordenación del territorio orientada a la captación, gestión y distribución de turbias (aguas pluviales) presentase una notable impronta territorial,

b. son unos ámbitos territoriales sujetos a fuertes dinámicas socio-territoriales y de urbanización que, en principio, incidirían en una intensificación en la circulación de los flujos de agua. El crecimiento urbanoresidencial registrado durante las últimas décadas, hasta el estallido de la crisis inmobiliaria de 2007, ha convertido parte de estas áreas en uno de los destinos turístico-residenciales más importantes de Europa con importantes repercusiones territoriales y ambientales provocadas por cambios masivos en los usos del suelo (Morote, 2014). Junto a esa expansión urbano-residencial también cobra protagonismo el fuerte crecimiento del poblamiento urbano disperso, que requiere dotaciones de agua de boca, pero también de riego para jardines (Morote y Hernández, 2014). Estas nuevas tipologías se asocian a una notable expansión de los usos turístico-residenciales (municipios litorales de las comarcas del Campo de Alicante y Vega Baja del Segura) o a la difusión urbana en un área metropolitana como Barcelona o la ciudad de Alicante.

c. se trata de un espacio sujeto a cambios importantes en la gobernanza del agua, como la privatización de los servicios de suministro (y parcialmente de los servicios de saneamiento) en los últimos años. En ciertas áreas, como la región metropolitana de Barcelona, cambios incipientes en el origen del agua utilizada en los hogares se traducen en variaciones de gobernanza del ciclo hidrosocial (March y Saurí, 2014). Por ejemplo, ciertos sistemas descentralizados de suministro de agua (aguas «grises» o aguas pluviales para aprovechamientos domésticos) pueden cambiar las relaciones de poder y control sobre los flujos urbanos de agua. En otros casos, el ciclo del agua incluye intercambios entre los sectores agrario y turístico, una variante poco habitual, pero muy interesante por lo que comporta en la gestión flexible, pactada y armonizada entre usuarios de recursos con calidades y funcionalidades distintas en una simbiosis que puede ejecutarse o no dentro del mercado como marco regulador (Rico et al., 2013). 
d. la arquitectura de los sistemas de abastecimiento a ciudades y espacios turísticos de estas regiones presenta una complejidad creciente alimentada por controversias relacionadas, por ejemplo, con los trasvases o la desalinización a gran escala. Sin embargo, las políticas de gestión de la demanda y del uso de recursos alternativos, particularmente la utilización de pluviales y residuales regeneradas, aunque cuenta con importantes e innovadoras iniciativas en algunos casos, han recibido mucha menos atención cuando se trata de las comunidades donde mayores esfuerzos se han hecho en los sistemas de abastecimiento.

e. el litoral mediterráneo español, y por ende estas zonas, también puede verse afectado por importantes variaciones climáticas con una incidencia directa en la disponibilidad física del agua y que requerirían cambios sustanciales en las fuentes de abastecimiento, potenciando el uso de recursos no convencionales.

EL USO DE RECURSOS DE AGUA NO CONVENCIONALES EN EL MARCO DE LAS POLÍTICAS DE SOSTENIBILIDAD Y EN EL CONTEXTO DEL CICLO HIDROSOCIAL DEL AGUA

La adaptación al cambio climático constituye uno de los retos principales de las sociedades a escala global (IPCC, 2014). En nuestro país, los efectos previstos del calentamiento térmico planetario en las condiciones climáticas se manifestarán, según los modelos predictivos, en una disminución del volumen de precipitaciones y un incremento en su variabilidad temporal y espacial (AEMET, 2015; Gallego et al., 2011). Algunas regiones, como las ribereñas del Mediterráneo van a resultar especialmente sensibles a los efectos de estos cambios en los patrones de precipitación (Benhamrouche y Martín Vide, 2012; Sauri et al., 2011), debido tanto a la escasez natural de lluvias como a la realidad de unas demandas de agua que se incrementaron de forma notable en las dos últimas décadas del siglo $\mathrm{xx}$, si bien, los consumos, al menos los urbanos, se han moderado e incluso reducido desde mediados de la pasada década (Gil, et al., 2015).

Estas tendencias se sitúan en el marco de la apuesta por una planificación y gestión de los recursos hídricos, en la que la fase histórica de las grandes infraestructuras hidráulicas destinadas a suministrar agua para una amplia variedad de usos superando limitaciones temporales y espaciales, parece hallarse en un proceso de cambio hacia un nuevo modelo en el cual los recursos hídricos se diversifican al tiempo que la gestión se inclina cada vez más hacia la demanda (Moral, 2009). Este nuevo periodo no es ajeno a recientes situaciones de sequía y de escasez de agua en el ámbito mediterráneo y peninsular, difíciles de afrontar si únicamente se cuenta con recursos convencionales. 
Ante escenarios mencionados de posible indigencia pluviométrica e hidrológica, la evaluación y eventual movilización de todos los recursos hídricos disponibles se erige como un objetivo social y territorial prioritario. En el ámbito urbano-turístico, de gran relevancia para la fachada oriental de la Península Ibérica, los recursos convencionales como el agua procedente de embalses y trasvases son en principio los más expuestos a precipitaciones más escasas y erráticas. Frente a ellos, el uso de recursos no convencionales se presenta como una alternativa de adaptación ante los nuevos escenarios climáticos de incertidumbre pluviométrica. Entendiendo como recursos no convencionales las opciones que representan para la gestión integrada de los recursos hídricos las aguas depuradas, las aguas pluviales y las generadas por desalación.

En los ámbitos de estudio, estos recursos no convencionales tienen como principal característica su versatilidad para un amplio abanico de usos en el ámbito urbano y turístico que no requieren una calidad equivalente a la del agua potable y se acomodan perfectamente al concepto de «fit for purpose» (recursos adecuados a usos específicos), de creciente protagonismo en la gestión integrada del agua. En este sentido, puede resultar muy difícil, por factores de disponibilidad y de coste, atender a todos los usos y demandas urbano-turísticas con agua potable, pero, en cambio, puede resultar mucho más factible si se desarrollan recursos con calidades ajustadas a cada uso. En este sentido, los recursos adecuados a usos específicos pueden contribuir decisivamente a aumentar la resiliencia territorial a episodios de incertidumbre hídrica generadas por el cambio climático. Su uso representaría un claro avance hacia un modelo de planificación y gestión de recursos hídricos más sostenibles y, sobre todo, resilientes.

Tanto para las aguas pluviales como para las aguas residuales, la literatura científica apunta hacia un claro cambio de paradigma puesto que de ser consideradas bajo la óptica del riesgo ambiental (inundaciones, contaminación, etc.) han pasado de manera progresiva a ser tratadas bajo la óptica de flujos que se pueden valorizar (Sedlak 2014; March et al., 2015). En el caso de las aguas pluviales y el drenaje urbano, los cambios en los usos del suelo generados durante las últimas décadas en los núcleos urbanos y turísticos del litoral Mediterráneo han provocado en muchos casos una desorganización y alternación de los colectores naturales que, junto con el incremento del sellado del suelo, han acabado por aumentar la frecuencia y gravedad de los episodios de inundación difusa. De ahí el fracaso en muchos casos de las estrategias de evacuación de estos flujos hacia los medios receptores y la promulgación de una serie de iniciativas de gestión de las aguas pluviales que van desde la construcción de grandes depósitos y tanques de retención de flujos derivados de precipitaciones de gran intensidad, hasta la creación de los denominados sistemas sostenibles de drenaje o SUDS, implicando cambios radicales en los procesos de urbanización, y el 
aprovechamiento de aguas pluviales en residencias privadas para usos como el riego del jardín.

En el caso de las aguas residuales urbanas, la mejora tecnológica en los sistemas de depuración, especialmente a partir de los tratamientos terciarios avanzados, que combinan ultrafiltración y desalinización, ha favorecido un aumento de los usos potenciales del agua regenerada. Desde hace dos décadas, las aguas regeneradas se utilizan para el regadío y para funciones ambientales en ámbitos como la Costa Brava en Gerona o áreas de la Comunidad Valenciana y la Región de Murcia. Sin embargo, su uso es menos frecuente en ámbitos urbanos aunque de manera incipiente figuran de manera destacada en algunas iniciativas municipales vinculadas con procesos de planificación territorial sostenible (Agendas 21 locales, Redes de municipios sostenibles, etc.).

En algunos casos, especialmente campings, es posible y especialmente viable la doble canalización de agua, no ya para utilizar el agua depurada o de pluviales por un lado y la potable por otro, sino aprovechando acuíferos propios salinizados no aptos para el consumo humano para las duchas, fregaderos y sanitarios, y el agua potable para beber y cocinar. Este sistema es posible, por ejemplo, en determinados sectores de las comarcas valencianas de La Marina Alta y La Safor, donde las precipitaciones son abundantes y el nivel freático es relativamente alto, pero donde las contaminaciones por intrusiones marinas imposibilitan el uso de los acuíferos propios para agua potable.

Por su parte, la desalinización ha sido la gran apuesta de la planificación hidrológica en nuestro país durante la última década (Programa AGUA), una vez descartadas las transferencias territoriales de agua a gran escala. La desalinización se está convirtiendo en una de las alternativas de mayor protagonismo en el mundo en relación al desarrollo de nuevos recursos hídricos, pero no se halla exenta de controversia (March et al., 2014; March, 2015).

El estudio de estos recursos alternativos debe incluir, asimismo, una dimensión social y política especialmente en lo que se refiere a las condiciones de gobernanza. En este sentido, el denominado enfoque de la ecología política urbana se ha erigido como un referente teórico de gran interés para examinar los procesos socio-ecológicos que ocurren en el interior de las áreas urbanas y turísticas y, particularmente, los marcos de poder bajo los que se metabolizan recursos como el agua. Así, flujos urbanos antes desconocidos, ignorados o tratados como peligrosos pueden pasar a adquirir nuevas funciones como recursos que pueden atraer el interés de ciertos actores como por, ejemplo, las compañías proveedoras de agua o grandes usuarios como las corporaciones locales (Troy, 2008). Trazar los flujos de pluviales a través del tejido urbano contribuye a explicar en toda su complejidad el funcionamiento de las ciudades modernas y su relación con los medios rurales y naturales más próximos. Como en el caso de los recursos convencionales, estos recursos no convencionales también 
forman lo que denominamos un ciclo hidrosocial. En las residuales y pluviales, está constituido por una compleja red de alcantarillado y colectores, bombeos, contadores, usuarios, regulaciones legales, estándares de calidad y, por supuesto, procesos físicos (precipitación, escorrentía, etc.). Desde la ecología política urbana se argumenta que los flujos de agua en las ciudades, incluyendo las desalinizadas, pluviales y las residuales depuradas, no dependen solamente de las condiciones climatológicas, hidrológicas y tecnológicas, sino también, y de manera muy relevante, de instituciones (formales e informales), de prácticas y de discursos; en otras palabras, de todo lo que constituye la gobernanza urbana del recurso (Swyngedouw, 2004).

Un recurso no convencional que puedes resultar interesante en determinados sectores y para fines muy concretos es el del agua de niebla, o, por mejor decir, el de la criptoprecipitación, en el que también deberíamos considerar al rocío nocturno. En determinados lugares del mundo la vegetación se ha adaptado a capturar este recurso ante las escasas precipitaciones convencionales. En ámbitos con precipitaciones escasas durante parte del año o todo él, pero con presencia de elevada nubosidad y humedad ambiental podemos encontrar vegetación morfológicamente adaptada a capturar por contacto las pequeñísimas gotas de agua que dejan rocíos y nieblas (Cereceda et al., 2004). En esos casos nos encontramos con paisajes vegetales sorprendentemente frondosos y con especies que, en teoría, necesitan una precipitación muy superior a la contabilizada por los pluviómetros tradicionales. La casuística es variada. Determinados sectores litorales desérticos del mundo, provocados por el fenómeno up-welling, no registran apenas precipitaciones convencionales. De hecho el Desierto de Atacama cuenta con medias anuales inferiores a $1 \mathrm{~mm}$ que, en realidad, suponen años enteros sin precipitación, 15 ó 20 en ocasiones, y precipitaciones puntuales cuando un Niño potente evita el fenómeno antes descrito, la subida de aguas frías a superficie, y da lugar a lluvias. En condiciones normales, en estos desiertos, el contacto entre el agua fría del mar y el aire cálido imposibilita casi por completo el ascenso en la columna de aire de la humedad y, por tanto, la condensación en altura. No obstante, ese mismo fenómeno sí propicia la aparición de numerosas nieblas, susceptibles de ser aprovechadas por cierto tipo de vegetación y, en su caso, también por el hombre, en pequeñas comunidades locales. En otros lugares, la persistencia de los húmedos vientos alisios chocando contra relieves de cierta entidad, pero sin poder ascender en la vertical, por la presencia de los anticiclones subtropicales, da lugar a la generación de nieblas de advección, a auténticos «mares de nubes» que se desarrollan en las caras bien expuestas a estos vientos y que suponen, en unas franjas de altitud concreta, un magnifico aporte de humedad, al que han sabido adaptarse cierto tipo de especies vegetales. La laurisilva, en los sectores más favorecidos por estas condiciones, en las Islas Canarias, es un ejemplo de esta adaptación. Estas nieblas mojadoras 
fueron aprovechadas por los guanches para, especialmente con el prácticamente extinto Garoe, el más famoso de los llamados «árboles fuente», concentrar esta lluvia horizontal y conducirla y canalizarla para su aprovechamiento en épocas de sequía. En otros lugares del mundo, como ciertos sectores montañosos de Omán, donde esta precipitación horizontal puede suponer más del $60 \%$ de la total, determinados aljibes llevan a cabo este aprovechamiento de forma ancestral con olivos y ciertos arbustos mediterráneos.

De los recursos no convencionales comentados en párrafos anteriores, en el epígrafe siguiente se analizará de forma más detallada las aguas pluviales $\mathrm{y}$, en menor medida, un uso concreto para el agua de niebla captada en determinadas ubicaciones de las cimas valencianas. No solo es importante conocer de manera sistemática el volumen, las características y los usos de los flujos de aguas pluviales en los ciclos hidrosociales de ciudades y espacios turísticos del litoral Mediterráneo, resulta también necesario explorar con detalle iniciativas específicas que puedan ser utilizadas como posibles modelos de valorización de estos recursos a escalas más amplias. El número y características de estas iniciativas es importante aunque las más interesantes y de mayor potencial probablemente sean: a) las que implican la sustitución a una escala apreciable de flujos de distintas características en ámbitos públicos y/o privados; b) las que suponen intercambios de flujos (con o sin mecanismos formales de mercado) entre usuarios, como, por ejemplo, usuarios agrícolas y usuarios urbano-turísticos; y c) las que generen espacios de descentralización en el uso de los recursos (como, por ejemplo, las pluviales en áreas residenciales con jardín). Estas tipologías se han abordado parcialmente en trabajos previos en las áreas de estudio (Rico et al., 2013; Ortuño et al., 2015; Domenech et al., 2013), pero nunca de una manera sistemática e integrada en los nuevos enfoques de gestión integrada de recursos hídricos bajo condiciones de incertidumbre climática y escenarios de sequía.

\section{EL USO DE PLUVIALES EN ENTORNOS URBANOS}

\section{Las aguas pluviales y los cambios en el ciclo hidrosocial de las ciudades}

El modelo urbano (compacto o disperso) ejerce una influencia muy significativa en el metabolismo del agua y, por tanto, en las características de los ciclos hidrosociales. La incorporación de las aguas pluviales en los entornos urbanos viene determinada por una serie de factores, a saber:

a. distintos episodios de estrés hídrico (sequías), que acentúan la incertidumbre sobre recursos convencionales y junto a ellos, la también mayor frecuencia de precipitaciones intensas e inundaciones (Olcina, 2009) que han determinado el interés por la gestión de las aguas pluviales para minimizar estos riegos (Olcina, 2013; Moral et al., 2014a), 
b. la crisis del paradigma hidráulico convencional basado en grandes obras hidráulicas como embalses y trasvases cuya manifestación quizá más evidente fue la suspensión del proyecto del trasvase del Ebro en 2004 (Moral y Sauri, 1999; Moral et al., 2014b). La conflictividad social y territorial asociada a los grandes proyectos hidráulicos más convencionales han promovido el desarrollo de nuevos recursos, muy especialmente la desalinización. No obstante, la irrupción de la desalinización como alternativa a estas obras $\mathrm{y}$, al mismo tiempo, su controvertida eficiencia por los elevados costes y la falta de demanda de agua desalinizada, capaz de generar situaciones de escasez relativa para ciertos sectores como la agricultura de regadío o las capas sociales urbanas más modestas han determinado la irrupción de nuevos recursos como son las aguas pluviales.

c. la expansión del poblamiento urbano-turísticos experimentado por todas las regiones de la fachada mediterránea peninsular desde mediados de la década de 1990 hasta el estallido de la crisis inmobiliaria y financiera de 2007 con cambios cuantitativos y cualitativos en los modelos residenciales (urbanismo disperso con nuevos equipamientos como jardines, piscinas, campos de golf, etc.) podría haber representado un aliciente para incrementar los usos de estos recursos alternativos (Burriel, 2008).

d. los costes crecientes del ciclo hidrológico en las ciudades y áreas turísticas que podrían quizá moderarse o atenuarse con una mayor apuesta por la reutilización de residuales regeneradas y de pluviales para ciertos usos, que en estos momentos son atendidos con agua potable.

e. la movilización de estos recursos a nivel municipal vendría influida por la adopción de programas de mejora ambiental mediante Agendas 21 locales, planes municipales de turismo, ayudas de fondos estructurales europeos, etc. Los recursos alternativos ocupan un lugar destacado en las estrategias de gestión integrada de los recursos hídricos, muchas veces junto con medidas de gestión de la demanda. Todo ello forma parte de la creciente ambientalización a nivel local manifestada con herramientas de auditoría y gestión de recursos como las denominadas Agendas 21 locales. Aunque de implantación y desarrollo desigual, muchos municipios del entorno mediterráneo disponen de Agendas 21 o de otros instrumentos parecidos en los que el aprovechamiento de aguas pluviales y aguas grises ocupa un lugar importante. Existe, por tanto, una correlación entre la mayor sensibilidad ambiental que demuestra la adopción de estos instrumentos y el uso de flujos alternativos de agua aunque en la práctica esta correlación deba matizarse por la escasa concreción de las medias de gestión en muchos casos. 
f. la generación de nuevos flujos puede deberse a acciones no estrictamente pensadas para tal finalidad sino para otros cometidos. Así ocurre con los tanques de anticontaminación y con otras estructuras de contención de aguas pluviales para evitar inundaciones producidas por precipitaciones de gran intensidad. Todos estos recursos, debidamente regenerados, sirven también para generar importantes volúmenes de agua que luego pueden ser utilizados para otras funciones.

\section{Estudio de casos}

El análisis de casos presentado en los párrafos sucesivos no responde a un examen exhaustivo de todas las iniciativas desarrolladas en los ámbitos de estudio analizados, sino a una primera aproximación a un proceso de incorporación de los recursos pluviales al ciclo hidrosocial de determinadas ciudades.

El primero de estos estudios es el que corresponde al área metropolitana de Barcelona. En Octubre de 2002, el municipio de Sant Cugat del Vallès (Barcelona) aprobó la primera ordenanza de ahorro de agua de Cataluña y del estado. Esta ordenanza, promovida en el marco de la Agenda 21 Local del municipio, establecía como requisito para las viviendas de nueva construcción o grandes rehabilitaciones la incorporación de dispositivos de ahorro de agua y el uso de recursos alternativos como las denominadas aguas grises y las aguas pluviales. En Diciembre de 2005, el grupo de Recursos Hídricos de la Red de Ciudades y Pueblos para la Sostenibilidad de la Diputación de Barcelona elaboró una Ordenanza Marco para el ahorro de agua y fomento de recursos alternativos a escala local que serviría como modelo para los municipios que quisieran desarrollar este tipo de instrumento normativo. A finales de 2013, un total de 55 municipios catalanes (algunos de ellos situados fuera de la provincia de Barcelona) con más de 1'3 millones de habitantes en conjunto (17\% de la población de Cataluña) habían aprobado ordenanzas de ahorro de agua siguiendo y adaptando a sus necesidades la Ordenanza marco de la Diputación de Barcelona.

De los 55 municipios con ordenanza de ahorro de agua, 53 contemplan el aprovechamiento de aguas pluviales, que se erige como la medida favorita una vez el Código Técnico de la Edificación español (2006) y el Decreto de Ecoeficiencia de la Generalidad de Cataluña (2006) hubieran convertido en obligatorios los mecanismos de ahorro en lavabos, duchas, baños y cisternas de inodoro. El aprovechamiento de agua de lluvia ha tenido una acogida especialmente buena en los municipios con tipologías urbanas de baja densidad con jardín.

En la Ordenanza Marco de la Diputación de Barcelona, el uso de pluviales se sugiere como obligatorio en todos aquellos edificios (independientemente 
de su función principal) con más de $100 \mathrm{~m}^{2}$ de zona verde y opcional para las viviendas unifamiliares con una zona verde comprendida entre los 8 y los 100 $\mathrm{m}^{2} \mathrm{o}$ con una piscina de superficie superior a los $30 \mathrm{~m}^{2}$. Algunas ordenanzas como la de Sant Cugat del Vallés establecen que deben definirse los usos para los que se prevé recurrir a agua de lluvia, así como el volumen mínimo del depósito o tanque, mientras que en la Ordenanza Marco de la Diputación de Barcelona el tamaño del tanque o depósito se establece con volúmenes diferentes según el tipo de edificio, aunque para usos domésticos en viviendas unifamiliares raramente supera los 15.000 litros de capacidad. Sin que exista ninguna fórmula universal para calcular el volumen de los depósitos de agua pluviales se recomienda tener en cuenta la pluviometría media del área, el tamaño y materiales dominantes en la superficie de captación y la demanda de agua (riego del jardín, cisternas de inodoros, etc.). En la mayor parte de los casos, el tratamiento es relativamente simple y consiste en derivar fuera del sistema los primeros flujos de la precipitación, cuya calidad puedes ser muy mediocre, y colocar un filtro en la entrada del depósito. En algunos casos se puede añadir también algún desinfectante. Por ello, aunque los costes de instalación pueden resultar relativamente elevados, los costes de mantenimiento tienden a ser bajos. El periodo de amortización puede reducirse considerablemente a raíz del fuerte aumento de los precios y sobre todo de los impuestos vinculados al ciclo del agua. En Cataluña, la tributación progresiva del denominado Canon del Agua, con un precio superior a los 4 euros/metro cúbico en el tramo de consumo superior a 18 metros cúbicos mensuales, implica que las aguas pluviales se conviertan en una alternativa económicamente muy interesante para los usuarios.

En la Región Metropolitana de Barcelona, se calcula que alrededor de un $36 \%$ de la demanda doméstica de agua se utiliza para el riego de jardines privados (hasta un 50\% durante los meses estivales), mientras que el 60\% de este último (y el 100\% si se dedicara a las cisternas de inodoros) podría ser satisfecho con agua de origen pluvial. Por último, cabe resaltar que en general, la aceptación social de este recurso alternativo es elevada, especialmente entre los usuarios directos. En una encuesta efectuada a distintos agentes sociales vinculados al ciclo hidrológico urbano del área de Barcelona (desde empresas privadas, hasta administraciones, grupos ecologistas, etc.) se halló que las aguas pluviales eran el recurso favorito de todos estos agentes con la significativa excepción de las empresas de suministro (Domènech et al., 2013). El único parámetro para el que las aguas pluviales obtienen una puntuación baja es el de seguridad del suministro por las lógicas oscilaciones de la precipitación en entornos mediterráneos.

El segundo de los estudios de caso es el llevado a cabo por el CEAM. El CEAM, Centro de Estudios Ambientales del Mediterráneo, ha desarrollado entre 2002 y 2004 un proyecto titulado El agua de niebla como input en el sistema hidrológico de la Comunidad Valenciana, financiado por la Secretaría de Estado 
de Política Científica del Ministerio de Ciencia y Tecnología, dirigido por María José Estrela. Su inclusión se relaciona con lo señalado en el epígrafe anterior y con la importancia probada de este fenómeno en ciertos tipos de vegetación y contando con los precedentes en estas investigaciones en Chile (Roberto, 1999), Venezuela, Omán, Madeira, etc. En un primer momento, se trataba de averiguar la cantidad de precipitación de lluvia horizontal que se podía registrar en algunos lugares de la vertiente mediterránea española, especialmente bien favorecidos por la presencia de estas nieblas de advección, de esta nubosidad baja potencialmente generadora de agua. El estudio previo estimaba que un 5\% de la precipitación podía tener este origen con carácter general. Evidentemente, las montañas litorales y prelitorales están especialmente bien dispuestas a la entrada de los vientos húmedos del primer y segundo cuadrante $\mathrm{y}$, si la inestabilidad en altitud no acompaña para la formación de lluvias convencionales, esta humedad puede quedar enganchada en ellas en forma de nubosidad baja. El fenómeno es conocido y fácil de observar, pero se trataba de averiguar cuánto suponía en milímetros esta precipitación invisible, que se dejaba notar en algunos casos por la presencia de especies vegetales con necesidades hídricas superiores a las teóricas. Para ello se eligieron un total de 24 observatorios en Cataluña, Comunidad Valenciana, Murcia y Almería, en su mayoría litorales o prelitorales, pero también algunos más interiores, a no más de $60 \mathrm{~km}$, en zonas relativamente bien abiertas a estos flujos húmedos. Un ingenioso pluviómetro de niebla, que permitía restar además la precipitación convencional, dio resultados sorprendentes, con volúmenes medios que en 2010 oscilaron entre los 600 y los 1.500 $\mathrm{mm}$ anuales de precipitación horizontal. Aún más llamativo fue el hecho de que esta precipitación era regular a lo largo del año, y que incluso era especialmente intensa en pleno verano, momento en que se advierte especialmente la falta de lluvia convencional (Estrela et al., 2010).

Una vez demostrado el potencial de esta lluvia, sólo quedaba buscar una forma de captarla y un fin al que destinarla, acorde con las cantidades recogidas y con el emplazamiento, en algunas cimas relevantes de las sierras valencianas. Esto se hizo en otro proyecto del CEAM, continuación lógica del anterior, con el título: Evaluación del agua de niebla en la restauración de áreas afectadas por incendios forestales, financiado por la Secretaría de Estado de Política Científica del Ministerio de Ciencia y Tecnología, dirigido también por María José Estrela, desarrollado entre 2005-2008. Sólo se desarrolló en la Sierra de Ayora (Valencia), donde unas telas de $18 \mathrm{~m}^{2}$, debidamente preparadas y orientadas a la recolección y a la conducción de unos tanques de almacenamiento, permitían almacenar esta precipitación. El uso que se le dio fue el riego localizado de determinadas repoblaciones forestales, aunque también se vio interesante su derivación a tanques de extinción de incendio en lugares poco accesibles y mal dotados de agua convencional (Estrela et al., 2009). Lo cierto es que 
el proyecto tiene un gran interés, siendo conscientes de las limitaciones que implica su emplazamiento, la limitada envergadura del despliegue de las mantas de captación y el agua obtenida, pero, sin duda, sería interesante desarrollarlo en más ubicaciones y aplicarlo de forma más general, aunque fuera sólo con los dos usos descritos.

Un tercer caso de estudio es el que se ha llevado a cabo en la ciudad de Alicante. En ella, las actuaciones, aunque de momento presentan un carácter puntual, responden a varias finalidades. En primer lugar, la captación de pluviales con la finalidad de reducir la contaminación de las aguas de escorrentía (tanque anticontaminación de San Gabriel), acumular pluviales para evitar daños asociados a inundaciones (Parque la Marjal en la playa de San Juan) y la utilización de aguas regeneradas para riego de jardines. Todas estas iniciativas han sido puestas en funcionamiento por la empresa suministradora de agua en la ciudad de Alicante (Aguas de Alicante) (Gil et al., 2015). El tanque anticontaminación de San Gabriel con una capacidad de $1,1 \mathrm{Hm}^{3}$ y el Parque la Marjal en la playa de San Juan, de $45.000 \mathrm{~m}^{3}$, tienen como finalidad evitar el colapso de las depuradoras en situaciones de precipitaciones de gran intensidad. A esta finalidad principal, se une su posibilidad de, una vez convenientemente tratadas, destinarlas a usos cuyos requerimientos de calidad no sean agua de boca. El uso de aguas regeneradas para el riego de jardines tanto públicos como privados ha registrado un notable incremento desde un volumen de $182.208 \mathrm{~m}^{3}$ en 2007 a casi un hectómetro cúbico en 2013.Los recursos son proporcionados por las líneas de terciario avanzado existentes en las depuradoras de Orgegia $\left(10.000 \mathrm{~m}^{3} /\right.$ día $)$ y Rincón de León $\left(2.000 \mathrm{~m}^{3}\right)$. En la Ciudad de Alicante, el mayor consumidor es el Ayuntamiento, con $587.357 \mathrm{~m}^{3}$ facturados en 2013, con más del $87 \%$ del recurso distribuido en ese año, lo que ha permitido sustituir el consumo de agua potable utilizada para el riego de parques y jardines por agua regenerada. En relación con el abastecimiento a particulares, los recursos son suministrados desde la EDAR de Monte Orgegia, que ha permitido extender la red de riego a la zona de Vistahermosa, donde proliferan los chalés con jardín y pequeños huertos. La red también ha llegado a la partida de La Condomina y las urbanizaciones de Alicante Golf. Un factor de demanda, que ha propiciado su expansión, es la gran diferencia de tarifas existentes entre el agua regenerada, que en 2013 se distribuía a $0,32 € / \mathrm{m}^{3}$, y el agua potable que, por ejemplo, para un consumo de $30 \mathrm{~m}^{3}$ por trimestre puede alcanzar un coste medio de $2,23 € /$ $\mathrm{m}^{3}$, con los respectivos cánones de conservación, alcantarillado y saneamiento, más los impuestos incluidos, que la empresa suministradora debe repercutir a sus clientes. 


\section{CONCLUSIONES}

El análisis de los planteamientos teóricos en torno a los cuales se argumenta la necesidad de implementar el uso de pluviales en ámbitos urbanos y el análisis de estudios de caso pone de manifiesto una realidad dual tanto desde el punto de vista del grado de implementación como de su distribución territorial.

Uno de los primeros resultados es la escasa adopción en aquellos ámbitos semiáridos donde este sistema tuvo una amplia difusión territorial en el pasado. El análisis de las Agendas 21 Locales refleja la importancia de la adopción de prácticas sostenibles, pero generalmente éstas se refieren a la necesidad de aminorar los consumos mediante la adopción de sistemas de mayor eficiencia en grifería y electrodomésticos o en algunos casos a la necesidad de reutilizar el agua de piscinas de hoteles para el baldeo de calles como sucede, por ejemplo, en Benidorm. En ninguna de manera explícita se hace referencia a la captación y reutilización de pluviales. La realización de encuestas a propietarios de viviendas en urbanizaciones con usos exteriores (piscinas y jardines) bien particulares o colectivos en 9 municipios del litoral alicantino para conocer las estrategias que adoptaban en estos espacios para reducir los consumos de agua pusieron de manifiesto que éstas se sintetizaban en gran medida en la reducción de las superficies ajardinadas y en la sustitución de especies por otras con menores requerimientos hídricos. La opción vinculada a la recolección de agua pluvial y destinarla, posteriormente, al riego de su jardín no había sido aplicada en el $99 \%$ de los entrevistados, pero tampoco era una cuestión que se planteasen a medio plazo.

En cambio, el aprovechamiento de aguas pluviales en los municipios catalanes que han promulgado una ordenanza de ahorro de agua representa una de las alternativas de mayor aceptación y de impacto potencial más elevado especialmente en lo que se refiere a los usos exteriores. Esta aceptación concuerda con la observada en otros países como, por ejemplo, Australia (Hurlimann, 2009) y tiene mucho que ver con la autonomía que supone para los usuarios disponer de un recurso generado en sus propios hogares $y$, en la medida que precios e impuestos del agua de red sigan aumentando y con la reducción de costes económicos para los usuarios.

En conjunto, pues, las aguas pluviales han mutado hacia aprovechamientos urbanos siguiendo la lógica del desarrollo socioeconómico y territorial reciente, con el abandono de las prácticas agrarias tradicionales (y de su rico patrimonio paisajístico) que utilizaban el recurso y la emergencia de nuevos usos urbanos como el riego de jardines. En la medida que el cambio climático va a extremar todavía más el ciclo hidrológico en el Mediterráneo, las aguas pluviales, especialmente las derivadas de episodios de gran intensidad, deben pasar de riesgo a recurso. 


\section{BiBLIOGRAFÍA}

Aemet (Agencia Estatal de Meteorología) (2015). Proyecciones Climáticas para el siglo XXI en España. http://www.aemet.es/es/serviciosclimaticos/cambio_climat.

Benhamrouche, A. y Martín Vide, F.J.(2012). Avances metodológicos en el análisis de la concentración diaria de la precipitación en la España peninsular. Anales de Geografía de la Universidad Complutense 32(1), 11-27.

Box Amorós, M. (1995). Un aprovechamiento tradicional de agua en el Sureste Ibérico: los aljibes. Investigaciones Geográficas 13, 91-106.

Burriel, E. (2008). La década prodigiosa del urbanismo español (1997-2006). Scripta Nova 270 (64). Disponible en: http://www.ub.es/geocrit/sn/sn-270/sn-270-64.htm

Cereceda, P, Pinto, R., Larrain, H., Osses, P. y Farias, M. (2004). Geographical Description of Three Fog Ecosystems in the Atacama Coastal Desert of Chile. Third International Conference on Fog, Fog Collection and Dew. Ciudad del Cabo, Sudáfrica. Disponible en http://www.geo.puc.cl/observatorio/cereceda/C37.pdf

Clabsa. Clavegueram de Barcelona S.A. (2004): «Dipòsits de regulació d'aigües pluvials de la Ciutat de Barcelona». Página Web: www.clabsa.es/CAT/DipositsBCN. asp.

Estrela, M. J., Valiente, J. A., Corell, D., Fuentes, D., and Valdecantos, A., (2009). Prospective use of collected fog water in the restoration of degraded burned areas under dry Mediterranean conditions. Agricultural and Forest Meteorology, 149, 1896-1906. Disponible en http://dx.doi.org/10.1016/j.agrformet.2009.06.016

Estrela, M. J., Valiente, J. A., Corell, D., and Azorin-Molina, C., (2010). Análisis de la variabilidad espacial de la captación pasiva de agua de niebla en relación a la estacionalidad y a los patrones de viento en una región mediterránea (Comunidad Valenciana)». En: Fernández García, F., Galán, E., and Cañada, R., (eds.). Clima, Ciudad y Ecosistemas. Publicaciones de la Asociación Española de Climatología (AEC) Serie A, n. ${ }^{\circ} 7$.

Domenech, L., March, H. y Sauri D. (2013). Degrowth initiatives in the urban water sector? A Social Multi-criteria Evaluation of non-conventional water alternatives in Metropolitan Barcelona. Journal of Cleaner Production 38, 44-55.

Gallego, M.C., Trigo, R.M., Vaquero, J.M., Brunet, M., García, J.A., Sigró, J. and Valente, M.A. (2011). Trends in frequency indices of daily precipitation over the Iberian Peninsula during the last century. Journal of Geophysical Research: Atmospheres (1984-2012) 116 (D2). DOI: 10.1029/2010JD014255

Generalitat Valenciana. (2003) Depósitos de agua del proyecto de infraestructuras de prevención de incendios forestales, PIIPVA 051, fase i. Actuación en los TT.MM de Castell de Castell, Vall de Laguart, Vall d'Ebo y Vall de Gallinera. Conselleria de Medi Ambient. Alicante.

Gil Olcina, A.; Hernández Hernández, M.; Morote Seguido, A.F.; Rico Amorós, A.M.; SAurí Pujol, D.; March Corbella, H. (2015). Tendencias del consumo de agua potable en la ciudad de Alicante y Área Metropolitana de Barcelona, 20072013. Hidraqua, Gestión Integral de Aguas de Levante S.A. y la Universidad de Alicante 
Hernández Hernández, M. (2009). El paisaje como seña de identidad territorial: valorización social y factores de desarrollo, ¿utopía o realidad?, Boletín de la Asociación de Geógrafos Españoles 49, 169-184.

Hernández Hernández, M. y Olcina Cantos, J. (2013). Paisajes culturales y patrimonio hidráulico en tierras valencianas. Claves identificativas y estado de la cuestión». Hermosilla Pla, J. (ed). Las galerías de agua en la región noroccidental de Túnez. Patrimonio hidráulico mediterráneo, Universitat de València, 9-19.

Hernández Hernández, M.; Moltó Mantero, E. (2010). La cultura del agua en ámbitos semiáridos: valores paisajísticos, ambientales y culturales». Leco Berrocal, F.; Pérez Diaz, A.; Mateos Rodríguez, B.; García Marín, R.; Alberdi Nieves, V. (coords.). Territorio, paisaje y patrimonio rural. XV Coloquio de Geografía Rural, Universidad de Extremadura. Servicio de Publicaciones, 398-409.

Hernández Hernández, M. y Morales Gil, A. (2013). Los aprovechamientos tradicionales de las aguas de turbias en los piedemontes del sureste de la península Ibérica: estado actual en tierras alicantinas. Boletín de la Asociación de Geógrafos Españoles 63, 105-123.

Hurlimann, A. (2009). Household use of and satisfaction with alternative water sources in Victoria. Journal of Environmental Management 92 (10), 2691-2697. doi: 10.1016/j.jenvman.2011.06.007.

Intergovernmental Panel on Climate Change (2014). Climate Change 2013 and Climate Change 2014 (3 vols.) Disponible en : http://www.ipcc.ch/

López Gómez, A. (1951). Riegos y cultivos en la Huerta de Alicante. Estudios Geográficos 41,701-771.

Marco Molina, J. A. y Vera Rebollo, J. F. (1988). Impactos de los usos del suelo y erosión en las cuencas vertientes del sur del País Valenciano. Investigaciones Geográficas 6,7-32.

Marco Molina, J. A. y Morales Gil, A. (1995). Terrazas de cultivo abandonadas en el sureste peninsular. Investigaciones Geográficas 13, 81-90.

March, H. (2015). The politics, geography and economics of desalination: a critical review, WIREs Water 2, 231-243.

March, H.; Hernández, M. y SAuri, D. (2015). Percepción de recursos convencionales y no convencionales en Hreas sujetas a estrés hídrico: el caso de Alicante. Revista de Geografía Norte Grande 60, 153-172.

March, H. y SAURI, D. (2013). The unintended consequences of ecological modernization. Debt-induced reconfiguration of the water cycle in Barcelona. Environment and Planning A 45(9), 2064-2083

MARCH, H. y SAURí, D. (2014). Flujos de agua, flujos de capital: sistemas de abastecimiento y gobernanza del agua en Madrid y Barcelona. Investigaciones Geográficas 51, 7-2.

March, H.; SAURí, D. y Rico-Amorós, A.M. (2014). The end of scarcity? Water desalination as the new cornucopia for Mediterranean Spain. Journal of Hydrology 519, 2642-2652.

Martínez de Azagra, A. (1996). Diseño de sistemas de cosecha de agua para la repoblación forestal. Madrid, Mundi Prensa. 
Mogil Manso, J. y Martínez de Azagra, A. (2007). Técnicas de recolección de agua y de Oasificación para el desarrollo de la agricultura y la restauración forestal en regiones desfavorecidas. Cuadernos Geográficos 40, 67-80.

Moral ItuARTE, L. del (2009). Nuevas tendencias en la gestión del agua, ordenación del territorio e integración de políticas sectoriales. Scripta Nova 13, 281-309.

Moral Ituarte, L. del y Saurí, D. (1999). Changing Course. Water policy in Spain. Environment 41 (6), 12-36.

Moral Ituarte, L. del; Pita López, M. ${ }^{a}$ F.; Pedregal Mateos, B.; Hernández-Mora Zapata, N. y Limones Rodríguez, N. (2014a). Current paradigms in the management of water: resulting information needs. Progress in water geography. PanEuropean discourses, methods and practices of spatial water research. University of Tartu, 19-29.

Moral Ituarte, L. del; Pedregal Mateos, B.; Limones Rodríguez, N. y HernándezMora ZaPATA, Nuria (2014b). Nuevos paradigmas en la gestión de recursos y riesgos hídricos: datos e información necesarios para una gestión integrada del agua. Boletín de la Asociación de Geógrafos Españoles 65, 519-542.

Morote Seguido, A.F. (2014). Tipologías urbano-residenciales del litoral de Alicante: Repercusiones territoriales. Ciudad y Territorio. Estudios Territoriales XLVI (181), 431-443.

Morote Seguido, A.F. y Hernández Hernández, M. (2014). Jardines y urbanizaciones, nuevas naturalezas urbanas en el litoral de la provincia de Alicante. Documents d'Anàlisi Geogràfica 60 (3), 483-504.

Olcina Cantos, J. (2009). Cambio climático y riesgos climáticos en España. Investigaciones Geográficas 49, 197-220.

Olcina Cantos, J. (2013). Experiences in adapting to Climate Change and Climate Risk in Spain. Climate Change Adaptation in practice: from strategy development to implementation. Wiley-Blackwell, New Jersey, 253-268.

Moltó Mantero, E. y Hernández Hernández, M. (2012). La subexplotación de los paisajes culturales y su incidencia en los riesgos naturales. Una propuesta metodológica. Araque, E.; Baena, R.; Delgado, C. et ali (eds.). Investigando en Rural. Asociación de Geógrafos Españoles (AGE), 483-491.

MondeJAR SÁNCHEz, J. M. (2010). El riego de boqueras: una técnica hidráulica para la gestión ambiental de territorios semiáridos y lucha contra la desertificación. Aprovechamientos tradicionales de aguas de escorrentía en las cuencas de la comarca de l'Alacantí. Tesis doctoral. Inédita. Cortesía del autor.

Morales Gil, A. (1969). El riego con aguas de avenida en las laderas subáridas. Papeles del Departamento de Geografía 1, 167-183.

Morales Gil, A. y Box Amorós, M. (1986). El aprovechamiento del agua y los suelos en un dominio semiárido: la cuenca del barranco Blanco. Agost. (Alicante), Investigaciones geográficas 4, 7-24.

Ortuño, A., Hernández, M. y Civera, S (2015). Golf course irrigation and self-sufficiency water in Southern Spain, Land Use Policy 44, 10-18 
Rico, A.M., Saurí, D., Olcina, J. y Vera, J.F. (2013). Beyond megaprojects?. Water alternatives for mass tourism in coastal Mediterranean Spain, Water Resources Management 27, 553-565.

Roberto RomÁn, L. (1999). Obtención de agua potable por métodos no tradicionales. Obtención de agua a partir de las Camanchacas.Ciencia al Día Internacional 2(2). Disponible en http://www.ciencia.cl/CienciaAlDia/volumen2/numero2/articulos/ CADi-v2-n2-art2.PDF

Saurí, D., Olcina, J.; March, H.; Martín-vide, J.; Vera, F.; Padilla, E. y SerraLlobet, A. (2011). Case Study Mediterranean Coast of Spain. ESPON Climate: Climate Change and Territorial Effects on Regions and Local Economies. Applied research project 2012/1/4. Final Report. Annex 4. Disponible en: www.espon.eu/ export/sites/default/Documents/Projects/AppliedResearch/CLIMATE/ESPON Climate_Final_Report_Annex4_Spain_Case_Study.pdf

SEDLAK, D. (2014). Water 4.0. The past, present and future of the World's most vital resource. New Haven, Conn: Yale University Press.

Swyngedouw, E. (2004). Water and the Flow of Power. Oxford: Oxford University Press.

Troy, P. (ed) (2008). Troubled Waters. Confronting the Water Crisis in Australia's Cities. Canberra: The Australian national University Press. 